\title{
El paisaje como proceso de vida: experiencias de domesticación del bosque en el sur de Chile ${ }^{1}$
}

\author{
Piergiorgio Di Giminiani² y Martín Fonck ${ }^{3}$
}

\begin{abstract}
RESUMEN
Las ciencias sociales se han aproximado a la construcción del paisaje a partir de sus variables tangibles e intangibles, conceptualizándolo principalmente como una representación cultural o el reflejo de una ideología. Desarrollos analíticos recientes han indicado el carácter procesual del paisaje, enfatizando la importancia de la experiencia en la interacción social entre elementos humanos y no humanos. Para demostrar los alcances teóricos de una definición del paisaje como proceso de vida, se presenta el caso del paisaje colono en el sur de Chile, centrándose en el valor de la domesticación en la relación agencial entre habitantes y bosques. El análisis desarrollado en el presente artículo da cuenta de cómo, en contextos de colonización, el sentido de lugar y la pertenencia geográfica no se expresan a través de adscripciones identitarias tradicionales asociada a una presupuesta cultura campesina sino mediante la involucración de los seres humanos con los bosques, en la búsqueda por transformar espacios forestales salvajes en bosques domesticados.
\end{abstract}

Palabras clave: Paisaje, lugar, sociedad colona, Chile.

\begin{abstract}
Social sciences have approached the social construction of landscape through a focus on tangible and intangible variables, primarily conceptualized as a cultural representation or a reflection of ideology. New analytical developments have highlighted the procedural character of the landscape, emphasizing the importance of experience on social interaction between human and non-human components. In order to show the theoretical implications of the definition of the landscape as a life process, we present here a case study on the colonial landscape of southern Chile. In particular, we will focus on the value of domestication, characterizing the agential relation between forests and their inhabitants. Our analysis shows that, in the historical context of colonization, the sense of place and geographical belonging are not manifested through traditional identity claims associated with an assumed farming culture. Rather, they materialize as a result of human engagement with forests, aimed at the transformation of wild forest areas into domesticated woods.
\end{abstract}

Key words: Landscape, place, colonial society, Chile.

\footnotetext{
1 Los autores agradecen al proyecto Concurso Inicio 2012 Vicerrectoría de Investigación, Pontificia Universidad Católica de Chile y centro ICIIS (CONICYT) FONDAP/15110006), a nuestros colaboradores Daniela Jacob, Francisca de la Maza, Julián Moraga y a los participantes de este estudio en la comuna de Pucón, IX Región. Artículo recibido el 6 de abril de
}

2015, aceptado el 25 de mayo de 2015 y corregido el 26 de junio de 2015 .

2 Programa de Antropología, Pontificia Universidad Católica de Chile (Chile).E-mail: pdigiminia@uc.cl

3 Centro ICIIS, Pontificia Universidad Católica de Chile (Chile). E-mail: mfonck1@uc.cl 
Tradicionalmente el paisaje ha sido abordado por las ciencias sociales como una representación social naturalizada y estable del entorno físico. De este modo, se ha entendido como una porción de espacio caracterizada por elementos simbólicos o culturales (Sauer, 1963; Jackson, 1984); o desde un punto de vista histórico, como una imagen asociada a ideologías dominantes (Cosgrove, 1984; Williams, 2001). Sin embargo, su carácter estático y semiótico ha sido problematizado por un cuerpo amplio de literatura que ha enfatizado, bajo distintas premisas, el carácter procesual y experiencial del paisaje, destacando su profunda variabilidad cultural, la cual tambien expresa las formas particulares en que los habitantes interactúan con su entorno físico en distintos contextos (Hirsch, 1995; Ingold, 2000; Jackson, 1989; Massey, 2006; Relph, 1981; Tilley, 1994; Thrift, 2007). Nuestro objetivo es proponer una caracterización del paisaje como proceso de vida, que amplíe la definición de este fenómeno como idea y lo presente en tanto manifestación colectiva de valores ideológicos típicos del contexto geohistórico particular en que se produce. Repensarlo como proceso de vida nos permite superar dicotomías clásicas de conceptualizaciones que distinguen, por ejemplo, entre paisajes culturales y naturales, demostrando así que las significaciones humanas del paisaje no dependen de una simple acción hermenéutica direccionada a un espacio compuesto por elementos simbólicos, sino que son el resultado de distintos procesos socioecológicos (Low \& LawrenceZúñiga, 2003, Urquijo y Barrera, 2009).

Asimismo, buscaremos demostrar los alcances teóricos de la definición de paisaje como proceso de vida mediante un enfoque en los procesos socioecológicos particulares de la domesticación en contextos culturales colonos. El sur de Chile se ha caracterizado por el fuerte vínculo entre deforestación y presencia de pequeños agricultores descendientes de los primeros colonos que se instalaron en sectores cordilleranos de difícil acceso y con alta presencia de bosque nativo. En esta zona, el proceso de colonización causó profundos cambios en el paisaje y desplazamiento de la población mapuche. Se presentará un caso de estudio en la localidad rural de Coilaco, ubicada en la Región de La Araucanía, donde ha sido desarrollado el tra- bajo de campo a través de la recopilación de entrevistas, observaciones etnográficas y datos históricos concernientes al proceso de colonización del sector desde 1930. El análisis desarrollado en el presente artículo pretende demostrar que en contextos de colonización el sentido de lugar y la pertenencia geográfica (Casey, 2013; Feld \& Basso, 1996; Pile, 2010; Pocock, 2014) no se expresan a través de adscripciones identitarias tradicionales, como comunidad, sino mediante las relaciones agenciales entre el bosque y las actividades humanas, en la búsqueda por transformar espacios forestales salvajes (definidos comúnmente en el sector como "selva") en bosques domesticados. En este sentido, la caracterización del paisaje como proceso de vida no depende necesariamente de elementos patrimoniales, más bien se centra en la interacción social entre elementos humanos y no humanos del paisaje (Descola, 2013; Ingold, 2012), el bosque y las habitantes locales en el presente caso. La estructura del artículo se desarrollará sobre la base de cuatro secciones. En la primera presentaremos una revisión de las principales conceptualizaciones teóricas del paisaje para luego discutir las tendencias principales del estudio del paisaje en el sur de Chile. Posteriormente, aclararemos los desafíos metodológicos de nuestra investigación y los beneficios producto de aportes comunes entre aproximaciones propias de la geografía humana y la antropología. A seguir, mostraremos el análisis de las principales características del paisaje en nuestro caso de estudio para dilucidar cómo dichas características indican un sentido del lugar centrado en los valores culturales de la domesticación en la sociedad colona. Finalmente nos aventuraremos en considerar las implicancias de nuestras conclusiones sobre un caso específico para los debates sobre la relación entre lugar y poder en los procesos de formación histórica de los paisaje forestales en Chile y América Latina (Urquijo y Bocco, 2011; Bray et al., 2009; Camus y Solari, 2008; Donoso y Lara, 1995).

\section{Paisaje: representación, idea y proceso}

Cualquier definición fija y estable del concepto de paisaje parece estar destinada al fracaso debido a la heterogeneidad de usos 
y conceptualizaciones asociadas al término. En esta sección nos interesa destacar tres grandes tendencias en la conceptualización de paisaje a partir de tres términos puntuales: imagen, idea y proceso. Dichas definiciones tendrían los beneficios analíticos de ser aproximaciones que enfatizan cada una la relación entre los aspectos representativos, ideológicos o experienciales. Etimológicamente, las primeras nociones asociadas al concepto provienen de lenguas románicas o neolatinas relacionadas al latín pagus, que se refiere a alguna extensión de tierra cultivada, y pagensis, que corresponde a campestre y que derivó en paysage (francés), paesaggio (italiano) y paisaje (castellano), como lo afirma Tesser (2000: 20). De tal modo, queda señalado de manera clara en el origen del término un vínculo entre el territorio y los asentamientos humanos. Esta relación también se da en la traducción del concepto a las lenguas germánicas, donde la palabra alemana landschaft tiene derivaciones como landscape (inglés) y landschap (holandés) y con un claro paralelismo conceptual a través de la expresión land, la cual también contiene un sentido práctico del territorio (Cosgrove, 1984). Sin embargo, otro sentido asociado al término provendría del uso que comienza a darle la escuela flamenca de paisajistas a fines del siglo XVI en un sentido artístico, el cual comenzó a ser utilizado también en Inglaterra como una vista o panorama que podía captarse de una sola mirada desde un punto de observación (Tesser, 2000).

Desde una perspectiva histórica, el cambio en la concepción del espacio contenida en el concepto de paisaje puede ser vinculado al desarrollo del capitalismo en Europa en los inicios del incipiente mercantilismo italiano. El mundo cerrado del feudo comienza a ser reemplazado por una red compleja de relaciones centro-periferia que transforman concepciones espaciales, la cual ahora es construida desde los valores humanistas del Renacimiento (Lefebvre, 1991, Panofsky, 1970). Representaciones del paisaje estética y moralmente enriquecedoras reflejan los valores y a la vez el poder simbólicos de las nuevas clases mercantiles del centro de Italia. El paisaje es así un espectáculo ordenado y observado desde la ciudad hacia el campo. Como propone Denis Cosgrove, "el origen de la idea de paisaje en Occidente y su expresión artística han servido en parte para promover ideológicamente una aceptación de las relaciones de propiedad mientras que ha sostenido una imagen no alienada de la tierra como uso" $(1984: 64)^{4}$.

El concepto de paisaje como una representación objetiva del entorno físico introduce una nueva relación entre el ser humano como espectador y el mundo que puede ser representado como un espacio sistematizado (Panofski, 1970; Descola, 2013). Este ideal encuentra su expresión más evidente en el desarrollo de la perspectiva lineal comúnmente asociada a los avances matemáticos y teóricos del arquitecto Filippo Brunelleschi. Los desarrollos de la perspectiva guardan relación con una condición cognitiva del espacio que posiciona al ser humano como observador con un mayor énfasis en el individuo y su capacidad para modificar el mundo natural proyectando un orden geométrico hacia este (Cosgrove, 1984).

El paisaje como representación implica, desde un sentido epistemológico, objetificar lo subjetivo al instaurar
"una distancia entre el ser humano y el mundo, haciendo que la autonomía de las cosas dependa del ser humano; y estabili- za el universo externo a pesar de que en- trega la organización de esta exterioridad recién conquistada sujeta a un control total" (Descola, 2013:59-60).

Tal distinción también se encuentra presente en definiciones nominales de paisaje usadas por las aproximaciones clásicas en la geografía humana. Este es el caso de Carl Sauer (1963) que propone entender el paisaje cultural como resultado de expresiones humanas colectivas impuestas sobre el paisaje físico, generando una propuesta fundacional en oposición al determinismo ambiental. En este contexto, surge el paisaje como una forma estrictamente geográfica de pensar la cultura (Jackson, 1989). Sin embargo, las representaciones de paisaje en geografía y

\footnotetext{
4 Traducción propia.
} 
otras disciplinas han reproducido la idea del paisaje como un espacio caracterizado por una demarcación objetiva y un orden preestablecido, que consecuentemente existe de forma autónoma respecto a las experiencias y perspectivas del observador. El paisaje cultural, en última instancia, corresponde a su caracterización como imagen y por ende puede ser entendido mediante una aproximación interpretativa de tipo iconográfica entendiéndolo como un sistema de signos y símbolos a ser leídos como texto. En este sentido, tal como plantea Nogué, el paisaje es entendido como una proyección cultural de una sociedad en un espacio determinado, distinguiendo dos dimensiones intrínsecamente relacionadas, una física material, objetiva y una cultural y subjetiva (2008 y 2009). Dicha idea ha sido popularizada por autores como el historiador Simon Schama, quien propuso la interpretación del paisaje rural inglés como la materialización de una memoria colectiva popular (1996).

Desde la década de 1970, varias contribuciones de la geografía humana han propuesto una crítica al concepto de paisaje como una representación simbólica objetiva del entorno físico para enfatizar el aspecto ideológico e histórico del paisaje. Desde perspectivas distintas, los trabajos de Hoskins (1996), Williams (2001) y Lefebvre (1991) han sido claves para la conceptualización del paisaje como una forma de representar el espacio basada en los valores sociales hegemónicos de cada época. El paisaje es, en consecuencia, producido material y conceptualmente en base a distintas operaciones económicas. Cosgrove nos entrega una aproximación al paisaje como una idea según la cual este no es el mundo que percibimos sino la manera en que lo percibimos y representamos:

"La idea de paisaje representa una forma de ver, una forma en la cual algunos europeos se han representado el mundo a sí mismos y a los otros, y a través de la cual ellos han comentado sus relaciones sociales" $^{\prime 5}$ (1984:1).
Los paisajes serían entonces, de acuerdo a esta visión, una manifestación de ideologías típicas que justifican cambios materiales y a la vez determinan discursos que son representados en distintos campos de producción cultural. Por ejemplo, la celebración de la naturaleza salvaje que aparece por primera vez en el siglo XIX en la literatura $-\mathrm{y}$ distintos medios- está estrictamente vinculada a la expansión colonial de la época debido a que las imágenes dominantes de las nuevas colonias tienden a excluir a las poblaciones locales, un punto que se refleja en el principio legal de la terra nullius que sirvió para justificar la apropiación de tierra y la repartición entre colonos (Beinart \& Coates, 2002; Griffith \& Robins, 1997). En este sentido, la investigación sobre el paisaje tradicionalmente lo ha entendido como "una manera pictórica de representar, estructurar o simbolizar el entorno" 6 (Cosgrove \& Daniels, 1988:1). El paisaje como reflejo de ideales políticos dominantes nos permite desmentir la idea de que las representaciones del entorno físico sean actos neutrales desvinculados de otros intereses.

La definición del paisaje como forma de representar el mundo nos entrega diferentes posibilidades analíticas, en particular vinculadas a la relación entre ideología y representación del entorno físico. En este artículo, sin embargo, pretendemos ampliar dicha mirada sobre la noción de paisaje destacando su aspecto experiencial y procesual. La redefinición del paisaje como proceso de vida nace a partir de dos críticas hacia las dimensiones excluyentes contenidas en la definición de paisaje como idea: la primera hace referencia al hecho de que esta aproximación prioriza las representaciones artísticas y discursivas del paisaje por sobre los aspectos cotidianos de la significación del entorno físico; la segunda plantea que el paisaje visto de esta forma es independiente de las experiencias corporales desarrolladas en este. En otras palabras, se reproduce una dicotomía entre sujeto y mundo observado que se encuentra en las formulaciones tempranas del concepto de paisaje. Ambas críticas nacen desde el desarrollo teórico proveniente de una aproxima-

\footnotetext{
6 Traducción propia. 
ción fenomenológica al paisaje en distintas disciplinas a partir de los años ochenta (San Eugenio, 2014). El estudio fenomenológico del paisaje nace a partir de la influencia de Maurice Merleau-Ponty (1994), quien postula considerar el cuerpo propio (le corps propre) como el eje básico de la comprensión humana del espacio. Como plantea el mismo autor, "lejos de que mi cuerpo no sea para mí más que un fragmento de espacio, no habría espacio para mí si yo no tuviese cuerpo" (Merleau-Ponty, 1994:119). El quiebre de la dicotomía fija entre subjetividad y corporalidad presente en la obra de Merleau-Ponty puede ser así extendido al estudio del paisaje. Según la propuesta de Michel de Certeau (2000), el espacio se vuelve inteligible y significativo a través del uso de nuestro cuerpo, y en particular al relacionar entre sí elementos del entorno por medio de nuestros desplazamientos. El movimiento, en consecuencia, es en última instancia lo que permite la articulación de múltiples relatos de espacio. De acuerdo con De Certeau,

“el relato del espacio es en su grado mínimo una lengua hablada, es decir un sistema lingüístico distributivo de lugares en la medida en que se encuentra articulado mediante una 'focalización enunciadora', mediante el acto de practicarlo" (2000:142).

El surgimiento de un interés antropológico en el paisaje surge en los años noventa contemporáneamente con el creciente interés por la fenomenología en esta disciplina (Desjarlais \& Throop, 2011). A diferencia de aproximaciones al paisaje que enfatizan las experiencias subjetivas de la significación del lugar (Tuan, 1974), la antropología del paisaje ha enfatizado la relación entre paisaje como idea y experiencia, buscando superar el riesgo del solipsismo y reconocer la simultaneidad individual y colectiva del paisaje. La investigación antropológica del paisaje ha sugerido así que este no puede ser reducido ni a una aprehensión de signos y símbolos realizada por un observador independiente ni tampoco a las inscripciones significativas situadas en un fondo neutral fruto de una suma de experiencias individuales (Tilley, 1994; Hirsh, 1995; Bender, 2002; Ingold, 2000, Feld \& Basso, 1996). En otras palabras, el paisaje no es ni solamente inscrito como en el caso de las aproximaciones semióticas inherente al concepto de paisaje cultural, ni solamente incrustado. En esta línea la diversidad cultural del paisaje se presenta en las diferencias relativas a nuestras experiencias corporales en este, tal como afirma Casey: "Como se reúnen cuerpos en medio de ellos de manera profundamente enculturados, así las culturas se conjugan en circunstancias concretas de emplazamiento" (1996: 46). Bajo esta perspectiva, el paisaje emerge a raíz de la tensión entre una esfera idealizada, relativa a los valores estéticos y las representaciones sostenidas culturalmente, y una esfera donde las experiencias individuales se van incrustando en el entorno local, añadiéndose signos y significados al entorno físico. Como nos sugiere Hirsch, el paisaje entendido como proceso implica una tensión y un movimiento entre polos diferentes de la existencia, uno definido como primer plano efectivo que refiere a lo cotidiano, junto a las formas irreflexivas de la experiencia, y un segundo plano potencial que consiste en la representación e idealización de la vida humana; en otras palabras, "la manera que podríamos ser" (1995: 4).

Un modo en que el paisaje se materializa es a través del movimiento, el que permite a los humanos demarcar lugares por el trazo de límites y conexiones entre ellos (Ingold, 2000). Los caminos y las rutas establecidas constituyen una manera estructurada de relatar y significar el entorno físico, a pesar de que como individuos tenemos la capacidad de tomar caminos y rutas de forma personal (Rodman, 1992). El paisaje puede ser así definido como "una serie de localidades con nombres, una serie de lugares conectados por caminos, movimientos y narrativas" (Tilley, 1994: 34). Nuestros desplazamientos en el espacio no pueden ser considerados únicos debido a que ellos conectan recuerdos y símbolos colectivos y biográficos, permitiendo estructurar narrativamente el paisaje mismo. Una de las características primarias del paisaje es la materialización del tiempo percibido a través de experiencias incorporadas (Bender, 2002). El pasado expresado a través del paisaje nunca es estático y emerge como una consecución de múltiples situaciones que ocurren en distintos momentos y con ritmos diversos. Ingold define este conjunto como taskscape (2000: 194). Como tal, el paisaje nunca es simplemente una imagen del pa- 
sado, sino más bien el proceso a través del cual los cambios sociales y ambientales son experimentados, tomando así sentido. Si en las aproximaciones clásicas al concepto de paisaje los significados están inscritos en el mundo, en esta "Ios significados son recolectados del mundo mismo" (Ingold, 2000:192).

Desde la geografía humana latinoamericana, la construcción social del paisaje ha sido abordada con un énfasis en los procesos históricos de exclusión generados en la construcción y consolidación de la nación (Urquijo y Bocco, 2011; Hiernaux y Lindón, 2006; Lindón, 2007). Una atención particular se ha prestado a los procesos de colonización que han impactado en nociones comunes de paisaje entre distintos pueblos originarios caracterizadas por la presencias de cruces entre elementos topográficos políticos y religiosos (Christlieb y Zambrano, 2006). A raíz de los procesos de colonización se han generado imaginarios geográficos con el fin de denominar e incorporar áreas a los márgenes de los bordes nacionales. Estas áreas han sido homologadas a conceptos de espacios vacíos, de tierras hostiles o infértiles, invisibilizando de tal modo a sus efectivos ocupantes (Zusman, 2013). Un ejemplo emblemático es la Patagonia, que ha sido representada en la literatura y el arte con un énfasis en la idea de naturaleza indómita asociada al valor de masculinidad de los exploradores y colonos de la primera parte del siglo XX (Nouzeilles, 2002). Este artículo pretende contribuir a los debates de la geografía latinoamericana mediante una revisión de los procesos geográficos de colonización a partir de las experiencias medioambientales de habitantes locales insertados en trayectorias históricas de construcción de nación, como en el caso de los colonos. Hablar de paisaje como proceso de vida significa por lo tanto reconocer las implicancias de superar una visión patrimonialista del paisaje, basada en una división entre sus caracteres culturales y naturales, para poder entender la colonización como un proceso histórico inacabado, desigual y abierto a las experiencias afectivas de las personas involucradas en este.

Como hemos visto en esta sección, las recientes contribuciones teóricas sobre el paisaje enfatizan la tensión entre representaciones y experiencias como un proceso articulador del paisaje. Si bien el concepto de paisaje cultural se puede caracterizar por intenciones políticamente meritorias, como la defensa del patrimonio tangible e intangible asociado a ciertas localidades con características sociales únicas, analíticamente este concepto presenta una limitación fundamental en cuanto refuerza la oposición entre paisajes culturales y naturales, reduciendo la complejidad del fenómeno del paisaje a una cartografía de elementos patrimoniales. En esta mirada, ciertos grupos humanos poseerían paisajes culturales mientras que otros no, una idea que implica el desconocimiento del paisaje como una forma de entender la sociedad y la historia en cada contexto humano. Salir de una visión patrimonial del paisaje implica reconocer el carácter procesual de este y el rol que cumplen los elementos nohumanos en la atribución de significados a la experiencia humana misma. La propuesta del paisaje como proceso de vida pretende romper la dicotomía entre naturaleza y cultura al posicionar el concepto de vida como un proceso diacrónico que involucra tanto a seres humanos como otros agentes del paisaje. La definición del paisaje como proceso de vida no es por lo tanto una alternativa teórica a otras, como en el caso de las definiciones de paisaje como representación e idea. El desafío es poder repensar el paisaje desde un punto de vista procesual que reconozca tanto el potencial político de representaciones e ideas en forjar sentidos de pertenencia y exclusión geográfica, como los aspectos fenomenológicos y ontológicos de la involucración práctica entre humanos y elementos no humanos del paisaje. Como veremos a continuación, el paisaje colono nos puede entregar llaves interpretativas para elucidar la relación entre poder y lugar a partir del carácter procesual del paisaje, sin reducir este a un conjunto de elementos simbólicos tradicionales. Antes de presentar las principales características del paisaje colono emergentes fruto de la investigación, se vuelve necesario destacar las contribuciones existentes sobre el estudio del paisaje en el sur de Chile.

\section{Paisajes forestales y colonización en el sur de Chile}

Los estudios realizados sobre el paisaje en el sur de Chile han tenido un foco principal 
en las transformaciones medioambientales asociadas a los procesos de colonización en los últimos dos siglos (Camus y Solari, 2008; Camus y Hajek, 1998; Otero, 2006). Sin embargo, dichos estudios se caracterizan por poseer diferentes perspectivas que pueden ser categorizadas en tres grandes campos, a saber: los estudios ecológicos sobre cambios en el paisaje a partir de análisis cuantitativos sobre fragmentación de bosque (Armesto et al., 1994, Echeverría et al., 2006, Nahuelhual et al., 2012); estudios historiográficos sobre ocupación del territorio por parte de los colonos y empresas forestales (Camus y Solari 2008; Bengoa, 2000; Klubock, 2014; Clapp, 1995), y estudios antropológicos sobre los procesos interculturales de significación del paisaje relacionados con la cosmología mapuche (Di Giminiani, 2013, 2015a y 2015b; Dillehay, 2011; Skewes et al., 2014).

Las perspectivas ecológicas sobre el cambio en el paisaje han tenido un foco en la cuantificación del impacto humano hacia el bosque nativo, con un particular enfoque hacia los patrones de crecimiento y deforestación (Altamirano y Lara, 2010; Armesto et al., 1994). Mediante el análisis de imágenes satelitales, estos estudios han cuantificado las transformaciones del uso de suelo, señalando los principales patrones de la desforestación y fragmentación del boque, dando cuenta de cambios sustanciales en la configuración espacial del bosque nativo (Echeverría et al., 2006; Altamirano y Lara, 2010). Entre los aspectos más relevantes evidenciados por estos estudios se destaca el rápido crecimiento de especies no endémicas asociado con los desarrollos históricos de la industria forestal. Se ha demostrado por ejemplo que el pino insigne (Pinus radiata) de alto valor comercial pasó de aumentar de una extensión de 29.213 ha en 1975 (5,5\% del paisaje) a una de 224.716 ha en $2007,42,4 \%$ del paisaje (Nahuelhual et al., 2012).

Los estudios historiográficos sobre el proceso de colonización y transformación del medio ambiente en los últimos dos siglos se han enfocado en las prácticas sociales que han causado la deforestación y a la vez en los impactos sociales negativos de larga duración asociados a la pérdida de bosque nativo (Camus y Solari, 2008; Donoso y Lara, 1995; Otero, 2006). La extracción intensiva de madera y el uso de fuego para despejar áreas de cultivos han sido las primeras causas de deforestación (Willson \& Armesto, 2006). Este proceso se expandió entre las décadas del cincuenta y del setenta con el desarrollo de políticas estatales que pretendían fomentar el mercado transnacional de madera (Catalán y Ramos, 1999; Clapp, 1995). Los procesos de privatización de recursos naturales que caracterizaron las políticas neoliberales durante la dictadura militar en Chile (1973-1990) fueron particularmente intensos en el campo de la producción forestal. Gracias a las posibilidades entregadas por el Decreto Ley 701 de 1974, unas pocas empresas capitalizaron la gran mayoría de plantaciones forestales de especies endémicas que reemplazaron así terrenos previamente ocupados por la producción agrícola (Klubock, 2014). El rol público de estas empresas es fuertemente cuestionado debido a los efectos negativos sobre la calidad del suelo en las áreas con amplias extensiones de especies exóticas; la transformación de las condiciones de trabajo y demanda de fuerza laboral en comparación con las actividades agrícolas; y las disputas con organizaciones y asentamientos indígenas mapuches, quienes demandan la restitución histórica de terrenos expropiados en el pasado y hoy en manos de propietarios no indígenas.

El tercer campo de estudio del paisaje en el sur de Chile se refiere al análisis de vínculos y conceptualizaciones medioambientales en contextos interculturales con presencia de población no indígena y mapuche. En esta línea también se han desarrollado estudios sobre paisajes ceremoniales indígenas enfatizando la significación de los espacios naturales a través de procesos culturales. Por ejemplo, el arqueólogo Tom Dillehay ha dado cuenta de la importancia cultural y social que tienen los montículos de tierra conocidos como kuel en los usos ceremoniales indígenas (2011). En este sentido, junto al paisaje se articulan funciones sociales, económicas y religiosas, la cuales continuamente se van grabando en el paisaje, siendo los espacios ceremoniales vitales para su persistencia y continuidad. Otros estudios han destacado la importancia cultural de los recursos naturales, como agua o bosque, en la sociedad mapuche (Armesto et al., 2001; Di Giminiani, 2012; Skewes et al., 2014). 
La revisión de las principales tendencias en el estudio del paisaje en el sur de Chile revela que los aspectos culturales del paisaje entre la población colona han jugado un rol marginal en la producción académica. Con nuestra investigación esperamos posicionar el paisaje colono como un objeto de estudio que puede ser analizado mediante un enfoque en los aspectos culturales y experienciales. El desafío principal es el desarrollo de una metodología que permita destacar las principales características culturales de este tipo de paisaje sin reducirlo a una cartografía de elementos tradicionales y patrimoniales del entorno físico.

\section{Investigación: metodologías y contexto local}

Una definición de paisaje como proceso de vida requiere realizar un análisis que contemple tanto el carácter fenomenológico de la relación entre residentes locales y el entorno físico, como los discursos y simbolizaciones asociadas a los elementos topográficos. Por esta razón nuestra investigación adopta dos orientaciones metodológicas: por un lado, la recopilación de observaciones etnográficas con el fin de rescatar las experiencias de los habitantes locales; por otro, la realización de entrevistas semiestructuradas sobre historia y percepciones respecto a los cambios medioambientales. La etnografía, como metodología, propone en términos esenciales una ruptura entre la observación y la participación. Si bien tradicionalmente ha sido asociada a la antropología desde el rol fundacional de Bronislaw Malinowski en la primera mitad del siglo $X X$, distintas disciplinas han incorporado este método por su capacidad para rescatar experiencias individuales y compararlas de forma colectiva (Scott-Jones \& Watt, 2010). En geografía, los métodos etnográficos han sido utilizados principalmente para facilitar una comprensión local de los procesos de formación de paisajes, territorios y lugares (Herbert, 2000). La justificación epistemológica para la adopción de una metodología etnográfica se funda en el reconocimiento de que los procesos de formación del paisaje están íntimamente ligados a los lugares donde acontecen (Raaflaub \& Talbert, 2009). Estudiar etnográficamente el paisaje implica por ello reconocer que el entorno físico es comprendido por los actores a través de sus experiencias en el lugar. Como plantea Hirsch,

"está el paisaje que vemos inicialmente, y un paisaje que se produce mediante la práctica local y que podemos reconocer y comprender a través del trabajo de terreno y mediante descripciones etnográficas e interpretaciones"7 (1995: 2).

La etnografía permite una involucración con los ritmos y rutinas diarias constitutivas de los significados del paisaje, que Anderson (2004) define como coingredencia constitutiva, por la mutua implicación existente entre los significados atribuidos al entorno y los procesos cotidianos de significación. En nuestra investigación hemos acompañado a los participantes de este estudio en sus actividades cotidianas para representar de forma más sensible las distintas experiencias y conocimientos del paisaje local. El acto de caminar juntos en particular nos ha permitido una exposición no solo a las narrativas y representaciones de ciertos lugares, sino también a situaciones prácticas particulares en las cuales se asociaban ideas y narrativas a lugares específicos.

Las observaciones etnográficas han sido complementadas con entrevistas semiestructuradas en la misma área de estudio. Se han llevado a cabo 40 entrevistas con 21 habitantes de la localidad de Coilaco Alto, buscando captar la heterogeneidad de los diferentes habitantes del sector. Todas las entrevistas han sido estructuradas para permitir a los participantes de esta investigación flexibilidad respecto a sus temas de interés. Los principales temas discutidos en las entrevistas han sido sobre historia local, cambios en las prácticas agrícolas con particular énfasis en las actividades forestales, relación con núcleos urbanos cercanos, interacciones con instituciones públicas y relaciones interpersonales con vecinos del sector. En algunos casos las entrevistas han involucrado dos participantes o en otros se ha entrevistado al mismo participante tres veces. Esta técnica nos ha permitido profundizar ciertas temáticas que habían sido

\footnotetext{
7 Traducción propia.
} 
parcialmente discutidas por los entrevistados o conversar acerca de eventos acontecidos en el tiempo de la investigación. Para defender la privacidad de los participantes en este estudio hemos utilizado nombres ficticios.

El área de estudio corresponde al sector de Coilaco Alto en la comuna de Pucón, IX Región de Chile, Lat/Lon: $(-39.3,-71.7)^{8}$. Coilaco es un valle de 10 kilómetros atravesado por un río de nombre homónimo, situado en zona precordillerana. Su ecosistema se caracteriza por la presencia de roble hualle (Nothofagus obliqua) en las partes bajas del valle y coigües (Nothofagus dombeyi), además de lengas (Nothofagus pumilio) y ñirres (Nothofagus antarcticus) en las partes altas. Aparte de las especies arbóreas del género nothofagus es posible encontrar otros tipos árboles como el laurel, el arrayán y especies exóticas -en cantidades menores- como el Pinus radiata. En la toponimia local, Coilaco Alto corresponde a un sector rural compuesto por aproximadamente cien habitantes distribuidos en viviendas dispersas. Los residentes locales se dedican esencialmente a actividades ganaderas y forestales, sobre todo los que poseen más tierra. En general las extensiones de tierra fluctúan entre 20 y 40 hectáreas por familia, un promedio que, debido al carácter accidentado del terreno y los duros climas invernales, corresponde a pequeña propiedad en la definición institucional. La comuna de Pucón, donde se encuentra este sector, es uno de los principales centros turísticos de Chile. Sin embargo, el impacto económico de esta actividad ha sido contradictorio en la zona. Esta localidad se encuentra muy alejada de las principales atracciones turísticas del sector y solo en los últimos meses se han diseñado las primeras estrategias comerciales para fomentar el turismo. Consecuentemente, algunos residentes locales, en especial entre los más jóvenes, complementan sus ingresos con empleos de temporada en el pueblo de Pucón, ubicado a 30 kilómetros de distancia.

8 Este sector fue seleccionado dentro de un área amplia de estudio en la cual se encuentran distintos proyectos de conservación públicos y privados, y que los autores de este artículo están analizando en el marco de un proyecto de investigación sobre conceptos de sustentabilidad y acción ambiental en Chile.
A la vez, los residentes se sienten desaventajados económicamente por los altos precios de los servicios y bienes de su comuna.

\section{Paisaje colono y la domesticación del bosque}

Durante nuestra investigación logramos recopilar un cuerpo muy vasto de entrevistas y narrativas sobre la relación histórica entre bosques y habitantes locales de Coilaco Alto. Siendo imposible reportar con detalle este material en el marco del presente artículo, nos limitaremos a ilustrar los tres aspectos básicos del paisaje en Coilaco que se han evidenciados en nuestra investigación. Estos se refieren al rol del paisaje en articular la historia local, al uso de categorías locales referidas a distintos espacios forestales y a la significación de la acción humana con el entorno físico. En Coilaco las representaciones de paisaje y las memorias de la colonización se focalizan en la domesticación del bosque como una lucha histórica para hacer de este valle un hogar. Los relatos orales de los residentes locales sobre la historia del lugar enfatizan generalmente los logros de los primeros colonos, a los cuales están ligados genealógicamente, en transformar el valle de Coilaco en un espacio apto para la producción agrícola. Las primeras familias que se instalaron en este sector en la década de 1930 sin títulos de propiedad, lograron resolver su situación legal unos años después, tal como fue prescrito por el $N^{\circ} 2.087$ de 1908 (Correa et al., 2005: 40). A su vez, el proceso de conversión de matorrales y bosques en campos agrícolas hasta hoy es relatado con la expresión "limpiar los campos". Este concepto define los bosques como áreas inútiles para el desarrollo productivo de la actividad económica doméstica. Tras la expansión de la industria forestal a un nivel nacional en los años 60, los bosques comenzaron a convertirse en recursos económicos atractivos para los residentes locales. Muchos de ellos construyeron sus propios aserraderos o arrendaron porciones de sus propiedades a grandes compañías forestales. Pero el boom forestal en Coilaco fue de corta duración debido a la competitividad decreciente causada por la expansión del negocio agrícola después de los 70, provocando que la mayoría de los campesinos se centraran en actividades 
agrícolas. Solo unos pocos residentes en las partes altas del valle continuaron las prácticas comerciales de explotación forestal en forma permanente.

En Coilaco, cambios observables en bosques y campos agrícolas, y la presencia de caseríos y casas abandonadas son recuerdos convincentes del proceso inacabado y en movimiento de hacer de este valle un hogar. La historia humana en el valle está íntimamente relacionada con las formas en que el bosque crece y se retira y el esfuerzo de los colonos por mantener sus bosques "limpios". Una tarde, Juan, un hombre aproximadamente de cincuenta años, nos mostró cómo el paisaje en Coilaco puede revelar la historia del valle: "Este bosque estaba justo donde mi vecino solía vivir. Estaba todo limpio en ese entonces, había muchos cerezos y yo solía ir a recolectar frutos allí. Los Martínez se fueron y ahora es monte de nuevo". En el relato de Juan, los bosques limpios ejemplifican el valor del trabajo necesario para la domesticación de espacios salvajes, típico de muchas sociedades colonas (Dominy, 2001: 19). Durante la investigación, también fue posible registrar numerosos relatos en los que los jardines son valorados por su aspecto estético, siendo contrastados con categorías locales como "arbustos", "selva" o "matorrales", de tal modo, resaltando el rol de la agricultura en mantener espacios domesticados. Como ha sido observado en otros contextos rurales, las descripciones del paisaje en Coilaco Alto revelan un discurso de la moralidad que enfatiza el rol del trabajo duro y la aplicación del conocimiento para transformar terrenos silvestres en campos agrícolas (Lee, 2007: 93).

Durante el trabajo de campo caímos en cuenta de los diferentes significados que pueden ser asociados al término "bosque" o "selva" entre los colonos. La categoría de bosque es generalmente opuesta a la de selva y monte, vocablo referido a áreas empinadas e indómitas. Bosque corresponde a extensiones de tierras forestales que son propiedad de las familias locales y trabajadas por esta. Bosque en Coilaco es referido casi siempre en el genitivo, y solo extrañamente como una categoría local, como en frases sobre "la belleza del bosque". Una selva, en contraste, se considera como un espacio forestal fuera de la agencia e intenciones humanas. Esta es entendida como un sector periférico para los humanos, quienes ejercen poco control sobre su regeneración y crecimiento (Descola, 2013: 44). La historia en Coilaco está caracterizada por la transformación, en el movimiento que va desde la selva hacia el bosque, a través de la intervención humana, así como de la posibilidad opuesta de que el bosque se reconvierta en selva si cae en desuso. Elementos arquitectónicos dispersos en el paisaje son otras huellas mnemónicas presentes que permiten destacar la lucha histórica por la domesticación. En las sociedades colonas, los lugares asociados con eventos históricos de domesticación y fundación tienden a ocupar una posición central en los paisajes locales (Dominy, 2001; Strang, 1997). En Coilaco, las construcciones abandonadas, como casas y una escuela, le recuerdan a la gente sucesos del pasado en el lugar, así como el creciente fenómeno de la migración hacia los centros urbanos. En una de nuestras entrevistas, Ruby, una residente de aproximadamente 50 años, señala: "Los jóvenes se van y los mayores se mueren aquí. Ellos quieren vender la tierra. Todo esto volverá a ser selva". Esta cita ejemplifica una visión sobre el futuro compartida por muchos residentes locales, para quienes la vida humana en Coilaco es entendida como un momento precario en la lucha histórica por domesticar el bosque.

Los relatos históricos locales y las representaciones del paisaje en Coilaco se caracterizan por la presencia de categorías locales que vuelven evidentes el balance frágil entre domesticación humana del entorno físico y el fracaso del trabajo agrícola. Especialmente entre los residentes católicos más fervorosos, las ubicaciones de las montañas y bosques se consideran como resultado de la acción divina. Significativamente, la palabra "sembrador" es empleada con frecuencia para referirse a Dios en esta área. Mientras que la "selva" es un elemento primordial del paisaje, el bosque existe como consecuencia de la acción humana. Las representaciones del paisaje mediante juicios estéticos y narrativas personales en Coilaco, finalmente demuestran una comprensión de las actividades forestales no solo como prácticas económicas, sino también como una necesidad para asegurar la preservación del "bosque" en tanto espacio domesticado. 
Los residentes están conscientes de los riesgos severos traídos por la deforestación, que en general correlacionan con los decrecientes niveles de agua y el aumento en la frecuencia de sequías en las últimas décadas. Al momento, no existe evidencia empírica sobre deforestación y crecimiento forestal en el valle de Coilaco. Sin embargo, en la investigación se ha revelado cómo entre los habitantes de este sector uno puede encontrar estrategias informales de manejo forestal para lograr un equilibrio entre conservación forestal y necesidades económicas. Estas estrategias son generalmente conocidas mediante el concepto de "bosque limpio". Mantener un "bosque limpio" implica un proceso en movimiento de ir seleccionando árboles para ser cortados o preservados. Esta selección depende esencialmente de las categorías de árboles "buenos" y "malos", las cuales dependen del uso potencial que se le puede dar a la madera de cada ejemplar. Un árbol "bueno" es un recurso atractivo de madera para la construcción y comercialización, mientras que uno "malo" es destinado a leña para calefacción y actividades domésticas. Los árboles malos no son "malos" de acuerdo simplemente a la calidad de su madera, sino también porque compiten con los árboles "buenos" por los recursos de la tierra, lo que constituye una amenaza para la calidad general de la madera. La caracterización de un árbol como "bueno" o "malo" es lograda a través de la evaluación visual del tronco y el follaje en comparación con los árboles cercanos. Los árboles "buenos" son reconocidos por el grosor y la rectitud de sus troncos. La presencia de pigmentación en hojas también puede ayudar a los campesinos a detectar enfermedades no visibles a través del análisis de la corteza. El acto de mantener un "bosque limpio" no depende de una aplicación de esquemas preconcebidos sino de la ejecución de una serie de habilidades improvisadas que responden a cambios y modificaciones en el medio ambiente (Ingold, 2000: 147; Richards, 1993). Bajo el principio de selección informal y constante de árboles, la actividad maderera no es solamente ventajosa, sino necesaria en cuanto permite mantener domesticados los espacios forestales inestables y frágiles. Durante una entrevista, un participante en este estudio afirmo: "Los bosques requieren de nuestra intervención para existir. Si no, ¿cómo sería posible vivir? Esto sería todo selva".
Las observaciones etnográficas y las entrevistas recopiladas en Coilaco Alto demuestran una concepción local de paisaje caracterizable como colono, que se basa en la naturaleza agencial e interdependiente de bosques y humanos. Lejos de ser objetos de representaciones, los bosques en Coilaco están activamente involucrados en la estructuración y redefinición del espacio humano. El paisaje colono, en última instancia, se caracteriza por procesos de vida históricos en los cuales participan humanos y bosques de forma interdependiente. En este contexto, el crecimiento de los árboles y grupos locales es un proceso necesariamente continuo e interconectado (Rival, 1993: 648). Los principios del paisaje colono nos permiten también lograr una definición del concepto de lugar que pueda ir más allá de interpretaciones simbólicas y discursivas de la identidad, reconociendo la mutualidad de seres humanos y medio ambiente en la articulación de sentidos de pertenencia geográfica.

\section{Lugar y poder en el paisaje colono}

Por muchas décadas, la relación entre cultura y lugar ha sido un elemento central en las representaciones de grupos étnicos y sociales en las ciencias sociales. El origen del concepto de área cultural es generalmente atribuido a la antropología cultural norteamericana de la primera mitad del siglo XX, en particular al trabajo precursor de Franz Boas, cuyo objetivo principal era registrar los rasgos lingüísticos, religiosos y artísticos en común de distintos grupos étnicos localizados en grandes extensiones territoriales (Stocking, 1996). La estricta dimensión espacial de la cultura será posteriormente criticada en los años ochenta y noventa por numerosos autores que han propuesto una revisitación del concepto de cultura centrado en la movilidad de bienes, personas e ideas como condición intrínseca de la modernidad globalizada (Appadurai, 1988; Marcus, 1995). Aunque las ciencias sociales en las últimas tres décadas han reconocido la significancia de la cultura sin una necesaria condición de emplazamiento, el lugar sigue siendo una categoría geosociológica relevante. Si bien el lugar puede ser entendido como un concepto ordenador de nuestra inmersión y compenetración con 
el mundo (Pocock, 2014: 17) su importancia para comprender las distintas formas de pertenencia y subjetividad humana ya no se encuentra en la presencia de rasgos culturales en áreas delimitadas por bordes herméticos, sino en las diferentes formas discursivas y prácticas en las que se articulan distintos sentidos del lugar (Feld \& Basso, 1996).

Recientemente, la geografía humana ha redescubierto el concepto de lugar a partir del rol de los afectos y emociones en activar una ontología relacional, involucrando elementos íntimos y cercanos de nuestro entorno físico (Pile, 2010). La consistencia emotiva de los lugares coexiste con su carácter multifacético debido a que el mismo lugar puede ser cruzado por múltiples voces y significaciones sin que este pierda sentido para sus habitantes (Rodman, 1992). En esta visión, la importancia de los bordes no consiste en su impermeabilidad sino en su porosidad, siendo el movimiento entre lugares el proceso central para la articulación misma del sentido de lugar (Casey, 2013; Ingold, 2012). En concordancia con lo anterior, los lugares se generan mediante una articulación dialógica de alteridad, en cuanto basan su unicidad necesariamente en relación a otros.

Reconocer el carácter emotivo y experiencial del lugar no significa ignorar los procesos económicos y políticos tras la producción de los lugares. La formación de los lugares es un proceso profundamente asimétrico debido a la presencia de fenómenos que trascienden las experiencias de los mismos habitantes locales, los cuales juegan un papel crucial en la producción de los sentidos locales. La relación entre poder y lugar ha sido inicialmente desarrollada por Henri Lefebvre, quien nos invita a considerar el espacio como un producto social generado tanto por representaciones como por creaciones concretas dependientes de relaciones económicas contingentes (1991). En la misma línea argumentativa, distintas investigaciones han señalado el efecto determinante del mercado global en los sentidos de lugar (Escobar, 2001; Harvey, 2001; Massey, 2006; Soja, 1989). Como plantean Gupta y Ferguson, las experiencias aparentes de comunidad y de interacciones intersubjetivas son constituidas por relaciones espaciales de poder más amplias (1997: 7). El desafío analítico consiste en indagar la cons- trucción del lugar a partir de interacciones entre el carácter experiencial y los procesos globales de poder que lo constituyen. Esta aproximación permite reconocer las perspectivas locales de sus habitantes insertadas en redes políticas y económicas más amplias.

En geografía, como en otras ciencias sociales, el análisis del "bosque" ha surgido como tópico privilegiado para abordar tanto relaciones socioecológicas en contextos locales como procesos socioeconómicos globales. Distintas investigaciones sobre el uso y conservación de los recursos forestales han subrayado las complejas interacciones jerárquicas que involucran a habitantes locales y agentes externos, manifestando tensiones existentes entre conocimientos locales ecológicos y científicos, prácticas de producción local y mercado global, movimientos transnacionales de conservación y necesidades locales (Bray et al., 2009; Klooster, 2002; Tsing, 2005). En el caso del sur de Chile, las dinámicas del lugar se han relacionado históricamente al proceso de colonización de tierras mapuche y a la acumulación capitalista de recursos forestales, desde donde se han determinado desigualdades espaciales y marginalización de ciertas poblaciones. Sin embargo, una atención dada a las experiencias en el paisaje entendido como proceso de vida nos permite destacar que el sentido de lugar no puede ser disociado de la relación emplazada entre agentes humanos y no humanos del entorno físico.

Los resultados de nuestra investigación sobre el paisaje colono reafirman en gran medida las conceptualizaciones recientes sobre el carácter político del lugar y a la vez las expanden a través de las implicancias teóricas de la definición de paisaje como proceso de vida. La localidad de Coilaco Alto no es entendida por parte de sus habitantes como un conjunto de expresiones culturales homogéneas y delimitadas espacialmente. Sus habitantes no se consideran parte de una colectividad homogénea, pues manifestan visiones políticas, religiosas y culturales marcadamente heterogéneas. A su vez, la pertenencia a esta localidad es también profundamente dinámica, habiendo vínculos de amistad y parentesco muy estrechos entre habitantes locales y residentes de sectores cercanos, incluyendo asentamientos rurales indígenas mapu- 
ches. A pesar de esta heterogeneidad externa, Coilaco Alto, como muchas localidades del sector, es asociada a la expresión "comunidad campesina" por parte de visitantes, planificadores urbanos, profesionales del turismo y académicos. Esta tendencia claramente no es solo local, también se encuentra a nivel global. Por muchas décadas, las localidades rurales han sido tipificadas como comunidad, un concepto que presupone un modelo de colectividad orgánica y autárquica. Sin embargo, "comunidad", más que una categoría descriptivo-analítica, aparece como una proyección idealizada, propia de la condición moderna, hacia aquellas formas de vida social vistas en oposición a la vida urbana en contextos industriales (Cohen, 1985). Es así que la localidad de Coilaco Alto desmiente cualquier tipo de caracterización basada en una correspondencia mecánica entre cultura y lugar, así como también los atributos de una comunidad fija.

A pesar de la ausencia de una caracterización comunitaria, los habitantes locales expresan con frecuencia sus sentidos de pertenencia mediante categorías de autoidentificación articuladas dialógicamente con otras tipologías sociales (Tedlock y Mannhein 1995). En primera lugar, tienden a estar identificados y a autoidentificarse como "COlonos nacionales", una categoría que surgió durante los procesos de colonización de los territorios indígenas mapuches para distinguir entre colonos europeos, que llegaron gracias a empresas de colonización, y ciudadanos chilenos que se trasladaron al sur del país de forma autónoma. La diferencia entre colonos europeos y nacionales no solo habla de su procedencia, sino que expresa profundas divergencias socioeconómicas, pues los inmigrantes chilenos se instalaron en la zona sur con posterioridad a los inmigrantes europeos y fueron beneficiados con terrenos menos extensos y poco atractivos desde el punto de vista agrícola. Por esta razón la "autoidentificación" como colonos coincide con la de "campesino" y "pequeños agricultores", estableciendo así una diferencia con los grandes propietarios de tierra, comúnmente referidos como "dueños de fundo". Estas categorías de autoidentificación establecen una primera fuente de alteridad entre los colonos nacionales y los grandes propietarios de tierra que en muchos casos preservan apellidos de origen alemán y francés, producto de los procesos de colonización europea en el sector. La otra fuente de alteridad para los habitantes de Coilaco Alto es representada por los residentes mapuches, también conocidos como "paisanos" en la jerga local. A pesar de que las relaciones de amistad y parentesco son mucho más frecuentes con vecinos mapuches que con grandes agricultores, los residentes de Coilaco Alto reconocen evidentes diferencias culturales con las comunidades indígenas del sector, apuntando en particular a las prácticas religiosas mapuches, las cuales existen de forma complementaria a las prácticas religiosas comunes católicas y evangélicas ${ }^{9}$.

Resulta evidente que el sentido de pertenencia e identidad entre los habitantes de Coilaco se define por la oposición entre dos polos de alteridad: por un lado los grandes propietarios de tierra y por otro, los residentes mapuches. No obstante, el sentido de pertenencia en este sector no puede ser solamente explicado en términos sociológicos, es decir a raíz de relaciones grupales, sino que se necesita una interpretación socioecológica del sentido de lugar, incluyendo la relación entre humanos y componentes no humanos del paisaje. Vale decir, entre los habitantes de Coilaco su lugar es entendido temporalmente como una construcción histórica inestable, ya que muchos residentes temen el despoblamiento futuro de este sector causado por las dificultades de la pequeña silvicultura y la transformación de los "bosques" en selva. Repensar el concepto de lugar según las implicaciones del paisaje como proceso de vida significa reconocer que ningún lugar existe como una unidad socioecológica estable en el tiempo, sino que el lugar está en una constante y perpetua construcción. Hacer-lugar (place-making) en el caso del paisaje colono implica una constante necesidad de un tipo de domesticación que no imponga nuevos espacios antrópicos, sino la articulación de espacios naturales salvajes como lo vimos en los llamados "bosques limpios". La construcción constante del lugar asociada a la conceptualización del paisaje como proceso de vida depende a fin de cuentas de las diferentes perspectivas proyectadas

\footnotetext{
9 Comunidades indígenas son unidades que actúan como representantes legales de un grupo local de residentes indígenas.
} 
por distintos grupos -en este caso grandes propietarios de tierra, colonos nacionales y mapuches- sobre el futuro de su lugar y la relación entre paisaje y seres humanos. El proceso de hacer-lugar, sin embargo, no puede ser desvinculado de procesos políticoeconómicos globales. La inestabilidad de la pertenencia geográfica por parte de los residentes de Coilaco refleja desigualdades históricas en los procesos de domesticación de la naturaleza en el sur de Chile. La expansión agrocapitalista en los últimos 150 años en la zona sur del país, y en particular la concentración desigual del acceso a recursos naturales, ha generado un proceso de marginalización espacial doble: uno hacia la población indígena local, desplazada por la ocupación de sus territorios ocurrida a fines del siglo XIX, y otro hacia los colonos nacionales, excluidos de los beneficios de la colonización.

\section{Conclusión}

En este artículo, hemos analizado con particular atención la interdependencia entre bosque y habitantes locales en la conformación del paisaje colono, dando cuenta de categorías locales de significación de la acción humana en los espacios forestales. La conformación del paisaje involucra el bosque como un agente activo en la redefinición constante del espacio humano. En las perspectivas locales, la domesticación de selvas y bosques emerge no como un proceso de depredación sino como una actividad necesaria para la preservación del lugar mismo amenazado por los procesos migratorios de despoblamiento. El sentido de pertenencia geográfico se constituye desde la relación entre habitantes y elementos nohumanos del paisaje, dando cuenta de su carácter dinámico. Lejos de ser el paisaje un fenómeno simbólico y discursivo que representa una identidad particular, se trata más bien de un proceso donde los sentidos se configuran a través de relaciones socioecológicas,.

Nuestras conclusiones se insertan en el debate académico contingente sobre ontología y teoría no representacional en las ciencias sociales (Mol, 2002; Latour, 2005; Thrift, 2007). En dicho debate, distintos autores han demostrado el potencial que presentan los no humanos en enactar y redefinir las relaciones sociales. En este artículo, la extensión de la sociabilidad al campo no humano observa- da en el caso de estudio nos entrega nuevas claves para lograr una comprensión del paisaje que abandone una atención excesiva de sus aspectos patrimoniales, tradicionales y simbólicos, la cual excluye potencialmente aquellos elementos del paisaje que no reflejan las características generales de paisaje cultural. En este sentido, hablar del paisaje como proceso de vida nos permite reconocer el carácter transformativo, político y cultural de este fenómeno. En el caso de estudio presentado en este artículo, resulta evidente que el paisaje es tanto un proceso histórico externo al individuo que depende de procesos políticos y económicos globales, como un proceso intersubjetivo extendido a la esfera no humana del paisaje.

Finalmente, cobra relevancia señalar la potencialidad que ofrece el proceso etnográfico para generar -y discutir- nuevas aproximaciones al paisaje, con el fin de entender cómo el entorno físico es comprendido por los actores a través de su experiencia en el lugar. De tal modo, dicha metodología permite un acceso a las prácticas locales donde se configuran los sentidos cotidianos de lugar. Otra de las contribuciones que permite la presente metodología es comprender los procesos de formación del paisaje a través de los procesos de constitución de lugar. Sin embargo, como es evidenciado a lo largo de este artículo, existe un riesgo en considerar el lugar como una construcción únicamente local. La presente investigación así confirma la necesidad de abordar la discusión sobre el paisaje desde lentes disciplinarios diversos, atendiendo las diferentes escalas involucradas desde puentes temáticos y contribuciones metodológicas que permitan enfrentar los fenómenos desde su complejidad y diversidad.

\section{Referencias bibliográficas}

ALTAMIRANO, A. y LARA, A. Deforestación en ecosistemas templados de la precordillera andina del centro-sur de Chile. Bosque, 2010, No 31, p. 53-64.

ANDERSON, J. Talking whilst walking: a geographical archaeology of knowledge. Area, 2004, Vol. 36, № 3, p. 254-261.

APPADURAI, A. Introduction: Place and Voice in Anthropological Theory. Cultural Anthropology, 1988, № 3, p. 16-20. 
ARMESTO, J.; VILLAGRÁN, C. y DONO$\mathrm{SO}, \mathrm{C}$. Desde la era glacial a la industrial: La historia del bosque templado chileno. Ambiente y Desarrollo, 1994, Vol. 10, N 1, p. 66-72.

ARMESTO, J.J.; SMITH-RAMIREZ, C. \& ROZZI, R. Conservation strategies for biodiversity and indigenous people in Chilean forest ecosystems. Journal of the Royal Society of New Zealand, 2001, Vol. 31, Nº 4, p. 865877.

BEINART, W. \& COATES, P. Environment and History: The Taming of Nature in the USA and South Africa. London: Routledge, 2002.

BENDER, B. Time and landscape. Current Anthropology, 2002, Vol. 43 (S), p. 103-112

BENGOA, J. Historia del pueblo mapuche: siglos XIX y XX. Santiago de Chile: Lom Ediciones, 2000.

BRAY, D.B.; MERINO-PÉREZ, L. \& BARRY, D. (editors). The community forests of Mexico: Managing for sustainable landscapes. Austin: University of Texas Press, 2009.

CAMUS, P. y SOLARI, M.E. La invención de la selva austral. Bosques y tierras despejadas en la cuenca del río Valdivia (siglos XVI-XIX). Revista de Geografía Norte Grande, 2008, No 40, p. 5-22.

CAMUS, P. y HAJEK, E. Historia ambiental de Chile. Santiago de Chile: Pontificia Universidad Católica de Chile, 1998.

CASEY, E.S. How to Get from Space to Place in a Fairly Short Stretch of Time: Phenomenological Prolegomena. In: FELD, S. \& BASSO, K. (editors). Senses of place. Santa Fe: School of American Research Press, 1996.

CASEY, E.S. The fate of place. Berkeley: University of California Press, 2013.

CATALÁN, R. y RAMOS, R. Pueblo mapuche, bosque nativo y plantaciones forestales: las causas subyacentes de la deforestación en el Sur de Chile. Temuco: Ediciones Universidad Católica de Temuco, 1999.
CHRISTLIEB, F. y ZAMBRANO, A. Territorialidad y paisaje en el Altepetl del siglo XVI. México: Fondo de Cultura Económica, 2006.

CLAPP, R.A. Creating Competitive Advantage: Forest Policy as Industrial Policy in Chile. Economic Geography, 1995, Vol. 71, №3, p. 273-296.

CORREA, M.; MOLINA R. y YÁÑEZ, N. La reforma agraria y las tierras mapuches: Chile 1962-1975. Santiago de Chile: Lom ediciones, 2005.

COSGROVE, D.E. Social formation and Symbolic Landscape. London: Croom Helm, 1984.

COSGROVE, D.E. \& DANIELS, S. Introduction: iconography and landscape. In: COSGROVE, D. \& DANIELS, S. (editors). The Iconography of Landscape: Essays on the Symbolic Representation, Design and Use of Past Environments. Cambridge: Cambridge University Press, 1988.

DE CERTEAU, M. La invención de lo cotidiano. México: Universidad Iberoamericana, Departamento de Historia, 2000.

COHEN, A.P. Symbolic construction of community. London: Routledge, 1985.

DESCOLA, P. Beyond nature and culture. Chicago: Chicago University Press, 2013.

DESJARLAIS, R. \& THROOP, J.C. Phenomenological Approaches in Anthropology. Annual Review of Anthropology, 2011, $\mathrm{N}^{\circ}$ 40, p. 87-102.

DI GIMINIANI, P. Tierras ancestrales, disputas contemporáneas: Pertenencia y demandas territoriales en la sociedad Mapuche rural. Santiago de Chile: Ediciones UC, 2012.

DI GIMINIANI, P. The contested rewe: sacred sites, misunderstandings and ontological pluralism in Mapuche land negotiations. Journal of Royal Anthropological Institute, 2013. Vol. 19, No3, p. 527-544.

DI GIMINIANI, P. The becoming of ancestral land: place and property in Mapuche land 
claims. American Ethnologist, 2015a, Vol. 42, $\mathrm{N}^{\circ} 3$, p. 115-134.

DI GIMINIANI, P. Being-from-the-land: memory, self and the power of place in indigenous southern Chile. Ethnos, Journal of Anthropology, 2015b.

DOI:10.1080/00141844.2015.1028566.

DILLEHAY, T. Monumentos, imperios y resistencia en los Andes. Santiago de Chile: Ocho Libros Editores, 2011.

DOMINY, M.D. Calling the station home: Place and identity in New Zealand's high country. Lanham: Rowman \& Littlefield, 2001.

DONOSO, C. y LARA, A. Utilización de los bosques nativos en Chile: pasado, presente y futuro. En: ARMESTO J.; VILLAGRÁN, C. y ARROYO, M. (editores). Ecología de los bosques nativos de Chile. Santiago de Chile: Editorial Universitaria, 1995.

ECHEVERRÍA, C.; COOMES, D.; SALAS, J.; REY-BENAYAS, J.M. \& LARA, A. Rapid deforestation and fragmentation of Chilean temperate forests. Biological Conservation, 2006, No 130, p. 481-494.

ESCOBAR, A. Culture sits in places: Reflections on globalism and subaltern strategies of localization. Political Geography, 2001, Vol. 20, N², p.139-174.

FELD, S. y BASSO, K.H. (editors). Senses of Place. Santa Fe: School of American Research Press, 1996.

GRIFFITHS, T. \& ROBIN, L. Ecology and Empire: Environmental History of Settler Societies. Edinburgh: Keele University Press, 1997.

GUPTA, A. \& FERGUSON, J. Culture, power, place: Explorations in critical anthropology. In: GUPTA, A. \& FERGUSON, J (editors). Culture, power, place: ethnography at the end of an era. Culture, power, place: Explorations in critical anthropology. Durham: Duke University press, 1997.

HARVEY, D. Spaces of capital: Towards a critical geography. London: Routledge, 2001.
HERBERT, S. For Ethnography. Progress in Human Geography, 2000, Nº4, p. 550-568.

HIERNAUX, D. y LINDÓN, A. (editores). Tratado de geografía humana. México: Editorial Anthropos, 2006.

HIRSCH, E. Landscape: Between Place and Space. In: HIRSCH, E. \& O'HANLON, M. (editors). The Anthropology of Landscape: Perspectives on Place and Space. Oxford: Oxford University Press, 1995.

HOSKINS, W.G. The making of the English landscape. London: Penguin Books, 1996.

INGOLD, T. The perception of the environment: essays on livelihood, dwelling and skill. London: Routledge, 2000.

INGOLD, T. Being Alive: Essays on Movement, Knowledge and Description. London: Routledge, 2012.

JACKSON, J.B. Discovering the Vernacular Landscape. New Haven: Yale University Press, 1984.

JACKSON, P. Maps of Meaning. London: New York: Routledge, 1989.

KLUBOCK, T. La Frontera: Forests and Ecological Conflict in Chile's Frontier Territory. Durham: Duke University Press, 2014.

KLOOSTER, D. Towards Adaptive Community Forest Management: Integrating local forest knowledge with scientific forestry. Economic Geography, 2002, Vol. 78, № 1, p. 43-70.

LARA, A.; SOLARI, M.E.; PRIETO, M.R. y PEÑA, M.P. Reconstrucción de la cobertura de la vegetación y uso del suelo hacia 1550 y sus cambios en la ecorregión de los bosques valdivianos Iluviosos de Chile (35 $\left.5^{\circ}-43^{\circ} 30^{\prime} \mathrm{S}\right)$. Bosque, 201, Vol. 33, № 1, p.13-23,

LATOUR, B. Reassembling the Social: An Introduction to Actor-Network-Theory. Oxford: Oxford University Press, 2005.

LEE, J. Experiencing Landscape: Orkney Hill Land and Farming. Journal of Rural Studies, 2007, Vol. 23, № 1, p. 88-100, 
LEFEBVRE, $\mathrm{H}$. The production of space. Oxford: Basil Blackwell, 1991.

LINDON, A.; AGUILAR, M.Á. y HIERNAUX, D. (editores). Lugares e imaginarios en la metrópolis. México: Editorial Anthropos, 2006.

LOW, S.M. \& LAWRENCE-ZÚÑIGA, D. (editors). Anthropology of Space and Place: Locating Culture. Malden: Blackwell, 2003.

MARCUS, G.E. Ethnography in/of the World System: The Emergence of Multi-Sited Ethnography. Annual Review of Anthropology, 1995, No 24, p. 95-117.

MASSEY, D. Space, time and political responsibility in the midst of global inequality. Erdkunde, 2006, Vol. 2, № 60, p. 89-95.

MERLEAU-PONTY, M. Fenomenología de la percepción. Barcelona: Peninsula, 1994.

MOL, A. The body multiple: Ontology in medical practice. Duke: Duke University Press, 2002.

NAHUELHUAL, L.; CARMONA, A.; LARA, A.; ECHEVERRÍA, C. \& GONZÁLEZ, M.E. Land-cover change to forest plantations: Proximate causes and implications for the landscape in south-central Chile. Landscape and Urban Planning, 2012, № 107, p. 12-20.

NOUZEILLES, G. (editor). La naturaleza en disputa: retóricas del cuerpo y el paisaje en América Latina. Buenos Aires: Paidós, 2002.

NOGUÉ, J. (editor). La construcción social del paisaje. Madrid: Biblioteca Nueva, 2009.

NOGUÉ, J. El paisaje en la cultura contemporánea. Madrid: Biblioteca Nueva, 2008.

OTERO, L. La Huella del Fuego: Historia de los Bosques Nativos, Poblamiento y Cambios en el Paisaje del Sur de Chile. Santiago de Chile: Pehuén, 2006.

PANOFSKI, E. Meaning in the Visual Arts. Harmondsworth: Penguin Books, 1970.
PILE, S. Emotions and affect in recent human geography. Transactions of the Institute of British Geographers, 2010, Vol. 35, № 1, p. 5-20.

POCOCK, D. Introduction: imaginative literature and the geographer. In: POCOCK, D. (editor). Humanistic Geography and Literature: Essays on the Experience of Place. London: Routledge, 2014.

RAAFLAUB, K.A. \& TALBERT, R.J. (editors). Geography and ethnography: perceptions of the world in pre-modern societies. New York: John Wiley \& Sons, 2009.

RELPH, E.C. Rational landscape and humanistic geography. London: Croom Helm, 1981.

RICHARDS, P. Cultivation: knowledge or performance. In: HOLBART, M. (editor). An anthropological critique of development: The growth of ignorance. London: Routledge, 1993.

RIVAL, L. The Growth of Family Trees: Understanding Huaorani Perceptions of the Forest. Man, 1993, Vol. 28, № 4, p. 635-652.

RODMAN, M. Empowering place: Multilocality and multivocality. American Anthropologist, 1992, № 94, p. 640-656.

SAN EUGENIO, J. Approaches to the individual-landscape interaction as an evocation of intrapersonal communication. Convergencia, 2014, № 64, p. 13-38.

SAUER C.O. The Morphology of Landscape. In: LEIGHLY, J. Land and life: Selections from the writtings of Carl Ortwin Sauer. Berkeley: University of California Press, 1963.

SCHAMA, S. Landscape and memory. New York: Vintage, 1996.

SCOTT-JONES, J. \& WATT, S. (editors). Ethnography in social science practice. London: Routledge, 2010.

SKEWES, J.C.; GUERRA, D. y HENRÍQUEZ, C. Patrimonio y paisaje: dos formas de ensamblar naturaleza y cultura en la cuenca 
del río Valdivia, Sur de Chile. Chungará, 2014, Vol. 46, No 4, p. 651-668.

SOJA, E.W. Postmodern geographies: the reassertion of space in critical social theory. London: Verso, 1989.

STOCKING, G.W. (editor). Volksgeist as method and ethic: Essays on Boasian ethnography and the German anthropological tradition. Madison: University of Wisconsin Press, 1996.

STRANG, V. Uncommon Ground: Cultural landscapes and environmental values. Oxford and New York: Berg, 1997.

TEDLOCK, D. \& MANNHEIM, B. The Dialogic Emergence of Culture. Chicago: University of Illinois Press, 1995.

TESSER, C. Algunas reflexiones sobre el significado del paisaje para la geografía. Revista de Geografía Norte Grande, 2000, No 27, p. 19-26.

TILLEY, C. A phenomenology of landscape: Places, paths, and monuments. Oxford: Berg, 1994.

THRIFT, N. Non-representational theory: Space, Politics, Affect. London: Routledge, 2007.
TSING, A. L. Friction: An ethnography of global connection. Princeton: Princeton University Press, 2005.

TUAN, Y. Topophilia: a Study of environmental perception, attitudes and values. Englewood Cliffs: Prentice-Hall, 1974.

URQUIJO, P. y BARRERA, N. Historia y paisaje. Explorando un concepto geográfico monista. Andamios. Revista de Investigación Social, 2009, Vol. 5, № 10, p. 227-252.

URQUIJO, P. y BOCCO, G. Los estudios de paisaje y su importancia en México, 19702010. Journal of Latin American Geography, 2011, Vol. 10, N², p. 37-63.

ZUSMAN, P. La geografía histórica, la imaginación y los imaginarios geográficos. Revista de Geografía Norte Grande, 2013, № 54, p. 51-66.

WILLIAMS, R. El campo y la ciudad. Buenos Aires: Paidós, 2001.

WILLSON, M. F. \& ARMESTO, J.J. Is natural history really dead? Toward the rebirth of natural history. Revista Chilena de Historia Natural, 2006, No 79, p. 279-283. 